\title{
Bedside Transthoracic 2D Echo-Guided Blade Atrial Septostomy: First Reported Case in the Philippines
}

\author{
Dexter Eugene D. Cheng and Maycibel D. Magadaeg-Capero
}

Section of Pediatric Cardiology, Department of Pediatrics, College of Medicine and Philippine General Hospital, University of the Philippines Manila

\begin{abstract}
A nonrestrictive interatrial communication is mandatory in some patients with complex cyanotic heart disease. Up to the present time, Park blade atrial septostomy with a Park blade catheter is performed only under fluoroscopic guidance. We successfully performed a blade septostomy followed by balloon septoplasty monitored only under transthoracic 2-dimensional (2D) echocardiography (TTE), in a 7-week-old infant with dTransposition of Great Arteries (d-TGA), a restrictive patent foramen ovale (PFO), and small ventricular septal defect (VSD), who presented with severe hypoxemia. TTE confirmed the position of the blade before and during pullback to the right atrium, followed by balloon septoplasty. There was an adequate enlargement of the interatrial communication with subsequent improvement in the oxygenation of the infant. This is the first reported case of the performance of blade atrial septostomy as a bedside TTE guided procedure here in the Philippines.
\end{abstract}

Key Words: blade atrial septostomy, d-TGA, PFO, fluoroscopy, 2dimensional echocardiogram

\section{Introduction}

In patients with certain varieties of congenital heart malformations, an adequate interatrial opening (IAO) is essential for survival. An IAO is important in transposition of the great arteries (TGA), as well as for obligatory interatrial shunt lesions such as pulmonary atresia, tricuspid atresia and total anomalous pulmonary venous return. An adequate IAO is hemodynamically beneficial to patients with double-outlet right ventricle with a restrictive ventricular septal defect (VSD) and those with a univentricular heart and a unilateral restrictive atrioventricular valve. Balloon atrial septostomy (BAS), introduced by Rashkind and Miller in 1966, was the first innovative nonsurgical therapeutic modality in pediatric

\footnotetext{
Paper presented as poster and won 2nd place at the 18th ASEAN Congress of Cardiology, December 1-3, 2010, Waterfront Hotel Lahug, Cebu City, Philippines.
}

Corresponding author: Maycibel D. Macadaeg-Capero, MD Section of Pediatric Cardiology

Department of Pediatrics Philippine General Hospital

Taft Avenue, Manila 1000 Philippines

Telephone: +632 5548400 local 2125

Email: menggay_md@yahoo.com cardiac catheterization. Palliation has been achieved in the majority of patients who have undergone this procedure. ${ }^{1}$

In infants older than 1 month of age, and certainly for older children who might require an atrial septostomy for palliation of their cardiac defect, the atrial septum is too tough or thick for a simple BAS to tear the septum. In 1975, Park et. al. introduced the Park Blade Septostomy Catheter (Figure 1) (Cook, Inc., Bloomington, IN) and the blade atrial septostomy procedure to obviate this difficulty. The blade is opened carefully in the left atrium (LA) while it is continuosly observed under fluoroscopy. The blade septostomy is followed by a balloon septostomy or septoplasty. As a result of the combined blade and ballooning, equalization of pressures between the two atria as well as a measureable increase in the mixing of the systemic and pulmonary venous blood should occur. ${ }^{2}$

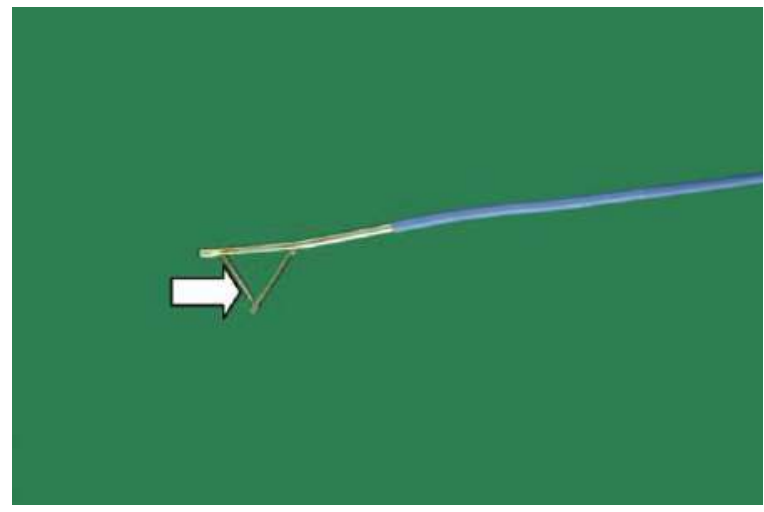

Figure 1. Park blade atrial septostomy catheter with the blade extended. (arrow shows the cutting blade side)

\section{Blade Atrial Septostomy Procedure ${ }^{3}$}

Blade atrial septostomy is performed when an adequately sized atrial communication is needed to enhance mixing at the atrial level or to decompress a chamber. The conditions for which there is general agreement that blade atrial septostomy is appropriate include infants older than 6 weeks with TGA who is not able to undergo arterial switch within 12 to 24 hours and in whom the atrial septum is unusually thick and may not be amenable to simple balloon atrial septostomy. 
The Park blade atrial septostomy catheter has a $2-\mathrm{cm}$ blade imbedded in the catheter and positioned across the PFO into the LA. The blade is opened once properly positioned in the LA. While pointing the blade anteriorly and to the left, the catheter is slowly withdrawn, thus cutting the lower margin of the PFO or atrial septum. This is repeated one or two times, varying the angle slightly, then followed by balloon septoplasty or septostomy.

\section{Case Report}

We report a 7-week-old male infant who was born full term via spontaneous vaginal delivery to a 19 year old G1P1 (1001) mother at home. No newborn care nor consults were done prior to this admission. He was rushed to the ER due to extreme cyanosis a few hours prior to admission. The infant was well nourished at $3.5 \mathrm{~kg}$, but was tachypneic and cyanotic with an oxygen saturation of $50 \%$. He was tachycardic with a Grade 2/6 systolic ejection murmur at the left mid-sternal border, and he had poor pulses. He was immediately intubated and his respiration supported. Chest radiograph showed no cardiomegaly, but with increased pulmonary vascular markings. The ECG was normal. Transthoracic Two-dimensional echocargiogram (TTE) showed d- Transposition of the great arteries (d-TGA), with a thickened and restrictive PFO measuring $2 \mathrm{~mm}$ (Figure 2), with maximum left to right shunt velocity of $1.6 \mathrm{~m} / \mathrm{sec}^{2}$. Concomitantly there was a small VSD and PDA.

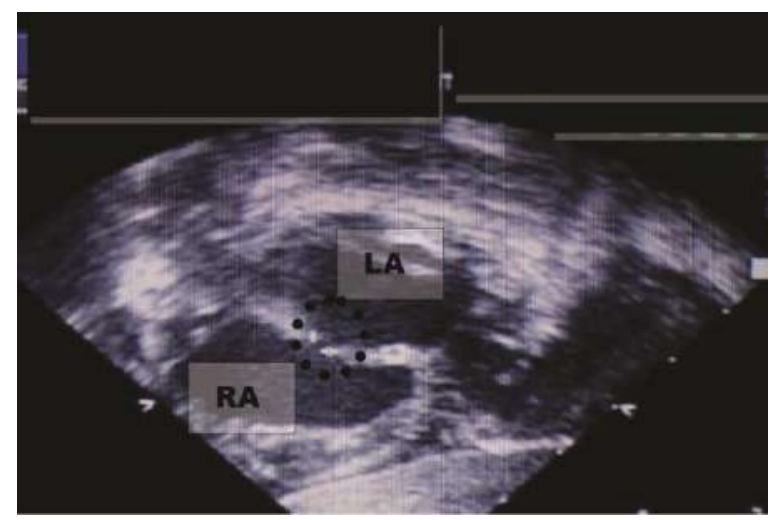

Figure 2. Subcostal long axis view of the atrial septum showing a PFO measuring $2 \mathrm{~mm}$. (RA- right atrium; LA- left atrium; dotted circle- PFO)

Due to logistical and financial constraints, the patient could not be brought to the cardiac catheterization laboratory for the Blade Septostomy. Thus, informed consent for an unorthodox bedside emergency blade atrial septostomy with balloon septoplasty was obtained after explaining the risks and benefits. The procedure was performed via standard technique with a $6 \mathrm{~F}$ long introducer sheath in the right femoral vein, a 4F JR catheter was used to cross the PFO and thus allow the introducer sheath to be positioned across the left atrium (LA). The location of the catheter was confirmed by TTE.

The Park blade catheter was deployed within the LA and was pulled across the atrial septum under echocardiographic guidance. The blade position was easily seen as it cuts through the atrial septum on echocardiography using the subcostal longhorn or 4 chamber views. The blade was retracted once it had crossed the septum (Figure 3). Several passes of blade across the PFO was done. The Park blade was removed and replaced with a $4 \mathrm{~F} 12 \mathrm{~mm} \times 3 \mathrm{~cm}$ Tyshak II catheter which was positioned across the atrial septum through the $6 \mathrm{~F}$ long delivery sheath. Atrial septoplasty was performed to further enlarge the IAO.

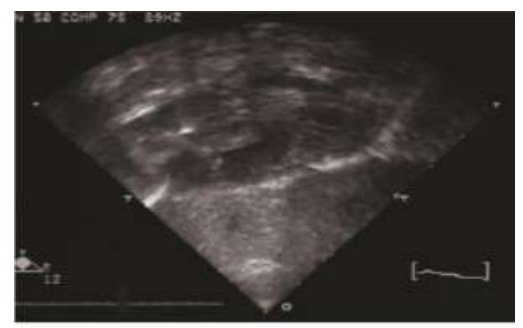

(a)

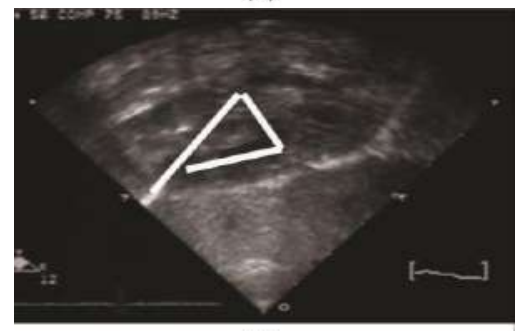

(b)

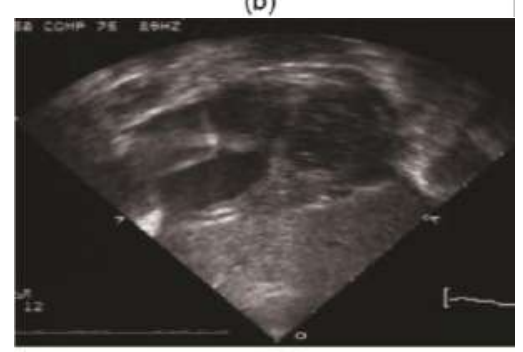

(c)

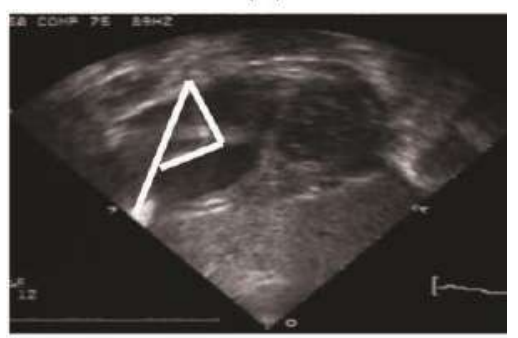

(d)

Figure 3. TEE guided monitoring of the blade (subcostal view)

(a) and (b).The Park blade catheter in the LA.

(c) and (d) The Park blade catheter as it was pulled across the atrial septum. 
Post-intervention, there was an adequate increase in the IAO to $5 \mathrm{~mm}$, with a decrease in the maximum velocity to 0.8 $\mathrm{m} / \mathrm{sec}^{2}$ (Figure 4). Oxygen saturations likewise improved to $85 \%$ from a baseline below $60 \%$. The patient was extubated within $24 \mathrm{hrs}$ following the procedure and was discharged on the $2^{\text {nd }}$ post-procedure day.

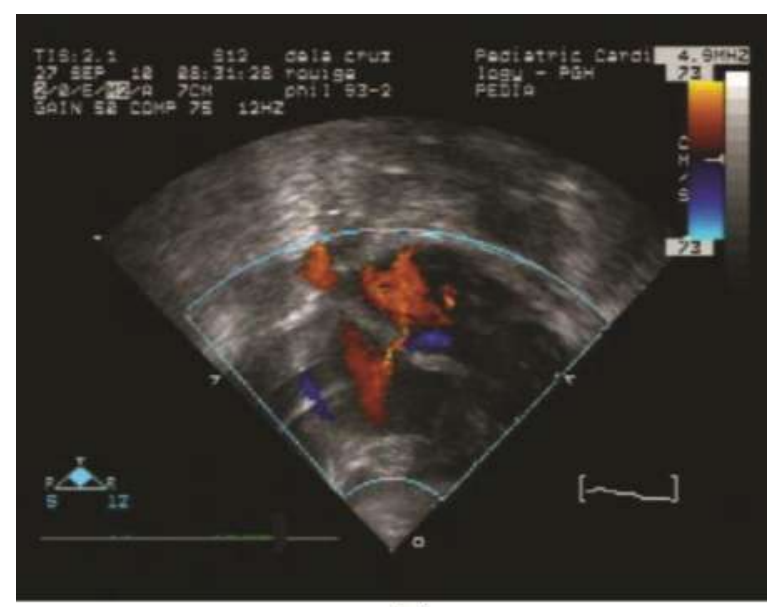

(a)

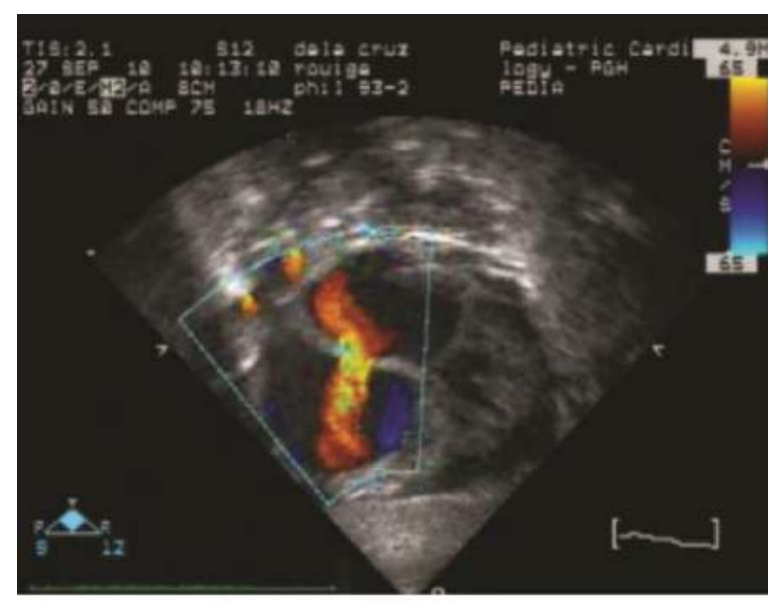

(b)

Figure 4. (a) Subcostal long axis view of the atrial septum showing the color flow across the IAO pre-blade atrial sepostomy. (b) Color flow across the IAO post-blade atrial septostomy.

\section{Discussion}

Rashkind Balloon atrial septostomy (BAS) remains an important palliative procedure in many infants with cyanotic congenital cardiac conditions before 2-3 weeks of age when the septum primum is thin. Femoral vein or umbilical vein, when available, is the common access used for this procedure. ${ }^{4}$

In 1966, the procedure was performed under fluoroscopic imaging. Recently it has become possible to perform septostomy under TTE. This shows clearly the intracardiac anatomy and the precise position of the catheter tip, which reduces the risk of inadvertently damaging the mitral valve or other intracardiac structures. The use of TTE to monitor the procedure has simplified this procedure dramatically; so that it can be performed at the bedside in the neonatal intensive care unit. ${ }^{4}$ Performing the bedside BAS avoids the transportation of severely ill patients. ${ }^{5}$ However, BAS has limitations in older infants due to the increased thickness of the atrial septum. In these cases, blade septostomy and static balloon dilation of the atrial septum are preferred to enlarge the interatrial communication. A collaborative study by Park et. al concluded that blade atrial septostomy is an effective means of enlarging an IAO without thoracotomy. ${ }^{6}$ It is best to be performed under biplane fluoroscopy.

In a study by Lin, et al., TTE was used as an imaging tool for the performance of BAS and blade atrial septostomy. Biplane fluoroscopy with 2-D echo or 2-D echo alone was used in the cardiac catheterization laboratory to place the balloon or blade septostomy catheter in the left atrium. Echocardiography confirmed the position of the septostomy catheter before pullback. Balloon inflation and balloon or blade pullback to the right atrium was performed with 2-D echocardiographic visualization. The safety and efficacy of 2-D echo to assist balloon or blade atrial septostomy in the catheterization laboratory or as the sole imaging device in the neonatal intensive care unit was confirmed. ${ }^{7}$ Blade atrial septostomy under TTE has also been applied successfully by Ozkutlu in 3 patients with TGA. ${ }^{8}$

In the local setting, not all cardiac catheterization laboratories are bi-plane capable. Furthermore, many patients are financially challenged and can not avail of these life saving procedures. With the same intent of transitioning the Rashkind procedure to the bedside, Blade atrial septostomy was offered to the family. Informed consent was obtained after stating that bedside blade has not been done locally, along with the expected risk and complications of the procedure.

The advantages of performing the blade septostomy as a bedside procedure are similar to those of Balloon Septostomy. TTE monitoring of Blade atrial septostomy avoids transporting severely ill patients and ionizing radiation. The bedside procedure costs less than half of that performed at the hemodynamics laboratory. ${ }^{8}$ The additional advantage of echocardiographic guidance is the fact that the blade could be easily seen as it touches the atrial septum, as it moves to cut the septum and exactly when it enters the RA. Precise manipulations of the blade under fluoroscopy are not possible since the atrial septum is not seen. Furthermore, due to the precise positioning, the blade could be moved in a slicing back and forth motion on the septum, which cannot be done under fluoroscopic guidance. Lastly, real time assessment of the effectiveness of the septostomy can also be done with 2D and color Doppler interrogation. ${ }^{9}$ 
In conclusion, the life-saving blade atrial septostomy procedure can be performed at the bedside under TTE for older infants safely and adequately. This is the first case performed and reported in the Philippines.

\section{References}

1. Rashkind WJ, Miller WW. Creation of an atrial septal defect without thoracotomy: palliative approach to complete transposition of the great arteries. JAMA. 196(11):991-2.

2. Moss and Adams' Heart Disease in Infants, Children, and Adolescents, $7^{\text {th }}$ ed vol 2, p70

3. Allen HD, Beekman RH 3rd, Garson A Jr, et al. Pediatric therapeutic cardiac catheterization: a statement for healthcare professionals from the Council on Cardiovascular Disease in the Young, American Heart Association. Circulation. 1998; 97(6); 609-25.
4. Padhi SS, Bakshi KD, Londhe AP. Balloon atrial septostomy through internal jugular vein in a 45-day-old child with transposition of great arteries. Ann Pediatr Cardiol. 2010; 3(1):77-9.

5. Marchi CH, Godoy MF, Santos MA, Moscardini AC, Hassen Sobrinho S, Croti UA. Echocardiographic monitoring of balloon atrial septostomy. Arq Bras Cardiol. 2005; 84(3):245-50.

6. Park SC, Neches WH, Mullins $\mathrm{CE}$, et al. Blade atrial septostomy: collaborative study. Circulation. 1982; 66(2):258-66.

7. Lin AE, TG Di Sessa TG, Williams RG. Balloon and blade atrial septostomy facilitated by two-dimensional echocardiography. Am J Cardiol. 1986; 57(4):273-7.

8. Ozkutlu S, Saraclar M. Superiority of echocardiographically assisted blade atrial septostomy. Jpn Heart J. 1992; 33(3):337-41.

9. Allan LD, Leanage R, Wainwright R, Joseph MC, Tynan M. Balloon atrial septostomy under two dimensional echocardiographic control. $\mathrm{Br}$ Heart J. 1982; 47(1):41-3

\section{Acta is now accepting} membership

Privileges of members:

- Allows you to submit articles for possible publication

- Have access to all the articles in the website (archives included) which can be downloaded and printed in pdf format

- Advertise your products/services in the available spaces of the website (for approval of the Editor-in-chief)

For details, please visit our website at www. actamedicaphilippina.com.ph or e-mail us at businessmanager@actamedicaphilippina.com.ph for any questions or queries. 